the men of Cadmus, but endowed with a different instinct.

Fortunately for us the public are comparatively ignorant of these our family secrets. Were the public aware that, from the want of some standard measure of medical qualification, no less than nineteen classes of medical men are in circulation-that a bishop can convert any biped into a doctor, even as the Roman despot would have converted his horse into a consul -it is evident that the current value of medical science would sink to the lowest point. Yet we cannot hope-indeed, we ought not to desire-that the public should remain ignorant much longer of the condition, as a body, of those to whom health and life are entrusted. Reform, then, we must have; for our own honor it is requisite; for the public good it is indispensable; in a word-to borrow an illustration from the present state of our monetary system-it is necessary that a new medical coin should be issued, of standard uniform weight and value, and current by law in every part of the United Kingdom.

Such, we repeat, is the earnest desire of a great majority of the medical profession. The peaceful but arduous labors of the medical man render peculiarly obnoxious to him the heart-burnings, jealousies, and strifes engendered by the present condition of medical affairs. 'To continue his way, quietly and respectably, in the exercise of his profession, benefitting mankind by his science, while he procures for himself and his family the means of honorable existence, is the main object of his ambition. To pursue this object in peace is impossible under the existing state of medical affairs. The conflicting interests of our rulers have entailed upon us the curse of the disobedient son; the hand of the medical man is incessantly raised against his brother; laws and regulations, with the enactment of which we have had no concern, have created a diversity of interests in our community, and introduced confusion and strife into a commonwealth where harmony of feeling and identity of interests should have prevailed; the character of the medical man is thus lowered in his own eyes, while the public estimation which he enjoyed is deteriorated in a still greater degree. These facts are brought home to every member of the profession in all parts of the empire: At one time the demand for medical reform was but a low and confused murmur; it is now the voice of the many-strong in number, and confident in the justice of their cause.

But what is the measure of reform which we ask? - what the amendment which the enemies of reform affect to represent as a destruction of ancient institutions, prompted by a vaguc and restless love of change? The majority of reformers - those with whom all prudent and true friends of the profession are prepared to act-require nothing very violent in action, or destructive in its effects; they simply ask, as a fundamental measure, the institution of an uniform qualification for all persons who shall hereafter enter the medical profession; and, as a necessary consequence, they demand an equality of privileges for all those who shall have thus obtained an uniform qualification.

We should like to hear a single argument against this moderate and reasonable demand, drawn either from general considerations or founded on the more narrow basis of expediency. It is useless for those who are opposed to all reform to evade the true question, and, by attacking the doctrines of a few visionaries, divert attention from the true grounds of debate between us. We do not pretend that those distinctions which have been established by time, or grown of their own accord out of the necessities of the public, should be abolished; we do not call for the destruction of institutions which have conferred many undoubted bencfits on the profession, if their existence can be rendered compatible with the public good; what we earnestly call for, on the part of our brethren, is a just and comprehensive arrangement, which shall embrace the whole profession, and put an end to the discord that now divides and distracts us.

\section{ROYAL MEDICAL AND CHIRURGICAL SOCIETY.}

Dr. Seymour, Vice-President, in the Chair.

Junc 14, 1842.

\section{A Case of Local Tubercular Deposit upon the Surface of the Brain. By Robert Dunn, Esq.}

The patient was a little boy, two years old, a fine intelligent child, who had been lealthy from the time of his birth. He had suffered little during dentition. At eleven months he had twelve teeth, and could then walk alone. On the 7th of October he was first seized, and he died in about six weeks-on the 15th of November. He had awoke in the morning as usual, and was suddenly scized with a jerking or convulsive twitching of the left hand, but which did not extend beyond the wrist. Excepting this continued convulsive jerking of the hand, the child seemed to be quite well. There were no indications of general derangement. About a fortnight before the child had fallen down stairs, and from that time had been irritable and fretful. In about twenty minutes the jerking subsided. It returned the next morning for half an hour, and then extended to the elbow. The following morning there was a slight attack; and the next day passed without any jerking; but there was partial paralysis of the hand and arm, pyrexia, and general constitutional disturbance. He complained of pain in the head, and frequently applied the hand to the right temple. He had been freely purged at first. Leeches were now applied ; counter-irritants ; cold lotions and ice to the head; saline medicine and calomel, and James's powder every four hours. This course was pursued throughout the disease; and the ung. hydrarg. fort. was also applied to the arm-pits night and morning, but salivation was not induced. During the next four 
or five days, he had frequent attacks of the convulsions, not confined to the hand and arm, but involving the whole of the left side and lower extremity in convulsive agitation, with twitchings of the eye and angle of the mouth, the attack lasting for hours. He cried, and even screamed violently, towards the close of the fits, but was sensible throughout, and could, at times, be soothed by his parents. The attacks werc followed by profound sleep for several hours, and the side was left partially paralysed. For about a week he had no return of the fits, except occasional jerkings of the hand and foot. The paralysis was not persistent. He was dull and heavy, sleeping many hours; yet sensible when awake, and eager for food. He had a quick but weak and irritable pulse; dry, hot skin; and great thirst. He was then seized with a kind of cramp or spasm in different parts of the affected side, arm, and leg. The pain was most distressing, and seemed, as in ordinary attacks of cramp-which it closely resembled-to be, in some degree, relieved by active friction. After suffering in this way for three or four days, he was left with decided symptoms of eflusion. The convulsions returned, attacking the right side in a similar manner in which the left had at first been affected. Both sides, and the whole body, indeed, eventually became affected with convulsive agitation, and the head, at the same time, drawn backwards. On the subsidence of one of these attacks he gradually sunk.

Note of the Post-mortem Appearances, by Dr. Todd, of King's College.-The scalp was pale and bloodless, like the rest of the body, which was much emaciated. The dura mater healthy. The vessels on the superfices of the brain were turgid with dark blood; but there was no sub-arachnoid effusion. The arachnoid cavity was natural. On the surface of the right hemisphere of the brain, under both the arachnoid and pia mater, there was a deposit of tubercular matter, disposed in patches of irregular shape and size, but the whole occupying a surface of about two inches square. The deposit was most abundant on the surface of the convolutions; but it nevertheless descended into the sulci between them, a circumstance which proved its connection with the deep surface of the pia mater. The cortical substance of the brain in contact with the tubercular matter was reddened, and greatly softened, and, on microscopic examination, evinced a nearly total destruction of the tubules in it, a great enlargement of the proper globules of the grey matter, and of the pigment granules which adhere to them. The softening extended a slight way into the subjacent white matter. On the edge of the left hemisphere, corresponding to the diseased patch of the right, a slight tubercular deposit had taken place in a similar manner, producing a red softening of the grey matter in contact, but not occupying more than half an inch square in surface. The ventricles contained more water than natural-about double-and did not collapse when laid open. The cercbral substance throughout, excepting at the diseased parts, was firmer than usual at the patient's age.

Mr. Dunn was of opinion that the fall which he had, had operated as an exciting cause in setting up diseased action about the tubercular deposit; and that the local affection-the simple twitching of the hand and jerking of the arm-was the consequence of the local membranous irritation thus induced. of the membranes and cineritious substance of the brain, he believed to be attended with convulsions, without decided or persistent paralysis, and that it requires the medullary matter to be involved to render the paralysis permanent. Admitting that red softening of the brain is the result of chronic inflammation of its substance, persistent paralysis, in the present case, was not to be expected until the inflammatory action had involved the medullary substance.

Notes of a Case of Petechial Cow-pox, with Observations on the Development of the Hamorrhagic Diathesis. By Dr. George Gregory.

A child was vaccinated at the Small-pox Hospital on the 19th of May, 1842; to all appearance in perfect health. On the 4th day, petechiæ were first seen. On the 8th day, extensive ecchymosis occupied the usual seat of areola, and the body was covered with petechial spots. On the 16th day, the scabs had began to drop off, and all hæmorrhagic appearances had subsided. Two children of the same family had been vaccinated at the same time from the same lymph, who both passed through the disease in a normal manner. The child had not exhibited any previous symptom of constitutional weakness.

The author considers this to have been a case of true petechial cow-pox ; not the coincidence of vaccination in an individual of hæmorrhagic tendency. $\mathrm{He}$ views it as an instance of the morbid matter of vaccine, usually so mild and so congenial to the human blood, proving poisonous to it, and developing the hœmorrhagic diathesis. It will be interesting, adds the author, to watch the future history of this child, to ascertain whether other morbid poisons have a like power of dissolving and deteriorating the quality of the blood, or whether this peculiarity attaches to the vaccine virus only.

The analogy subsisting between the phenomena of petechial cow-pox and petechial small-pox was noticed, and the frequency and severity of that form of variola illustrated by cases. The freedom of the brain and nervous system, in severe cases of petechial small-pox, was alluded to, which conspired with the present case in rendering it probable that the hæmorrhagic state is developed by a morbid poison through some direct agency on the blood itself, independent of the brain and nerves.

Petechial cow-pox is believed by the author to be exceedingly rare. He had never seen any other instance of it, and had only heard of two others.

\section{ACADEMY OF SCIENCES, PARIS.}

June 20.

\section{FALLOPIAN TUBES.}

M. Raciborski read a memoir on the connection between the Fallopian tubes and ovaries in the mammalia. As a general rule in domestic animals, the extremities of the tubes are arranged in such a manner as to envelope completely the ovaries during the breeding period.

In cases where the extremities of the tubes are not sufficiently large to embrace the whole ovary, the deficiency is supplied by prolongations of the peritoneum which form sacs embracing the ovary and fim- 